\title{
Properties of the solutions set for a class of nonlinear evolution inclusions with nonlocal conditions
}

Jingrui Zhang ${ }^{1 *}$, Yi Cheng ${ }^{2,3}$, Changqin Yuan ${ }^{2}$ and Fuzhong Cong ${ }^{2}$

\section{"Correspondence:}

ruierchat@yahoo.com.cn

${ }^{1}$ School of Aerospace Engineering,

Beijing Institute of Technology,

Beijing, 100081, People's Republic of

China

Full list of author information is

available at the end of the article

\begin{abstract}
In this paper, we consider the nonlocal problems for nonlinear first-order evolution inclusions in an evolution triple of spaces. Using techniques from multivalued analysis and fixed point theorems, we prove existence theorems of solutions for the cases of a convex and of a nonconvex valued perturbation term with nonlocal conditions. Also, we prove the existence of extremal solutions and a strong relaxation theorem. Some examples are presented to illustrate the results.
\end{abstract}

MSC: 34B15; 34B16; 37J40

Keywords: evolution inclusions; nonlocal conditions; Leray-Schauder alternative theorem; extremal solutions

\section{Introduction}

In this paper, we examine the following nonlinear nonlocal problem:

$$
\left\{\begin{array}{l}
\dot{x}+A(t, x)+B x \in F(t, x), \quad \text { a.e. } I=[0, T], \\
x(0)=\varphi(x),
\end{array}\right.
$$

where $A: I \times V \rightarrow V^{*}$ is a nonlinear map, $B: V \rightarrow V^{*}$ is a bounded linear map, $\varphi: H \rightarrow H$ is a continuous map and $F: I \times H \rightarrow 2^{V^{*}}$ is a multifunction to be given later. Concerning the function $\varphi$, appearing in the nonlocal condition, we mention here four remarkable cases covered by our general framework, i.e.:

- $\varphi(x)=x(T)$

- $\varphi(x)=-x(T)$;

- $\varphi(x)=\frac{1}{2 \pi} \int_{0}^{2 \pi} x(s) d s$

- $\varphi(x)=\sum_{i=1}^{n} \beta_{i} x\left(t_{i}\right)$, where $0<t_{1}<t_{2}<\cdots<t_{n}$ are arbitrary, but fixed and $\sum_{i=1}^{n}\left|\beta_{i}\right| \leq 1$.

Many authors have studied the nonlocal Cauchy problem because it has a better effect in the applications than the classical initial condition. We begin by mentioning some of the previous work done in the literature. As far as we know, this study was first considered by Byszewski. Byszewski and Lakshmikantham $[1,2]$ proved the existence and uniqueness of mild solutions for nonlocal semilinear differential equations when $F$ is a single-valued function satisfying Lipschitz-type conditions. The fully nonlinear case was considered by Aizicovici and Lee [3], Aizicovici and McKibben [4], Aizicovici and Staicu [5], GarcíaFalset [6], García-Falset and Reich [7], and Paicu and Vrabie [8]. All these studies were

\section{黛 Springer}

(c) 2013 Zhang et al.; licensee Springer. This is an Open Access article distributed under the terms of the Creative Commons Attribution License (http://creativecommons.org/licenses/by/2.0), which permits unrestricted use, distribution, and reproduction in any medium, provided the original work is properly cited. 
motivated by the practical interests of such nonlocal Cauchy problems. For example, the diffusion of a gas through a thin transparent tube is described by a parabolic equation subjected to a nonlocal initial condition very close to the one mentioned above, see [9]. For the nonlocal problems of evolution equations, in [10], Ntouyas and Tsamatos studied the case with compactness conditions. Subsequently, Byszewski and Akca [11] established the existence of a solution to functional-differential equations when the semigroup is compact and $\varphi$ is convex and compact on a given ball. In [12], Fu and Ezzinbi studied neutral functional-differential equations with nonlocal conditions. Benchohra and Ntouyas [13] discussed second-order differential equations under compact conditions. For more details on the nonlocal problem, we refer to the papers of [14-18] and the references therein.

It is worth mentioning that many of these documents assume that a nonlocal function meets certain conditions of compactness and $A$ is a strongly continuous semigroup of operators or accretive operators in studying the evolution equations or inclusions with nonlocal conditions. However, one may ask whether there are similar results without the assumption on the compactness or equicontinuity of the semigroup. This article will give a positive answer to this question. The works mentioned above mainly establish the existence of mild solutions for evolution equations or inclusions with nonlocal conditions. However, in the present paper, we consider the cases of a convex and of a nonconvex valued perturbation term in the evolution triple of spaces $\left(V \subset H \subset V^{*}\right)$. We assume the nonlinear time invariant operator $A$ to be monotone and the perturbation term to be multivalued, defined on $I \times H$ with values in $V^{*}$ (not in $H$ ). We will establish existence theorems of solutions for the cases of a convex and of a nonconvex valued perturbation term, which is new for nonlocal problems. Our approach will be based on the techniques and results of the theory of monotone operators, set-valued analysis and the Leray-Schauder fixed point theorem.

We pay attention to the existence of extreme solutions [19] that are not only the solutions of a system with a convexified right-hand side, but also they are solutions of the original system. We prove that, under appropriate hypotheses, such a solution set is dense and codense in the solution set of a system with a convexified right-hand side ('bang-bang' principle). Our results extend those of [20] and are similar to those of [21] in an infinite dimensional space. Furthermore, the process of our proofs is much shorter, and our conditions are more general. Finally, some examples are also given to illustrate the effectiveness of our results.

The paper is divided into five parts. In Section 2, we introduce some notations, definitions and needed results. In Section 3, we present some basic assumptions and main results, the proofs of the main results are given based on the Leray-Schauder alternative theorem. In Section 4, the existence of extremal solutions and a relaxation theorem are established. Finally, two examples are presented for our results in Section 5.

\section{Preliminaries}

In this section we recall some basic definitions and facts from multivalued analysis which we will need in what follows. For details, we refer to the books of $\mathrm{Hu}$ and Papageorgiou [22] and Zeidler [23]. Let $I=[0, T],(I, \Sigma)$ be the Lebesgue measurable space and $X$ be a separable Banach space. Denote

$$
P_{w(f) k c}(X)=\{A \subset X: \text { nonempty, weakly (closed) compact and convex }\} \text {. }
$$


Let $A \subset P_{f}(X), x \in X$, then the distance from $x$ to $A$ is given by $d(x, A)=\inf \{|x-a|: a \in A\}$. A multifunction $F: I \rightarrow P_{f}(X)$ is said to be measurable if and only if, for every $z \in X$, the function $t \rightarrow d(z, F(t))=\inf \{\|z-x\|: x \in F(t)\}$ is measurable. A multifunction $F: I \rightarrow$ $2^{X} \backslash\{\emptyset\}$ is said to be graph measurable if $\operatorname{Gr} F=\{(t, x): x \in F(t)\} \in \Sigma \times B(X)$ with $B(X)$ being the Borel $\sigma$-field of $X$. On $P_{f}(X)$ we can define a generalized metric, known in the literature as the 'Hausdorff metric', by setting

$$
h(A, B)=\max \left\{\sup _{a \in A} d(a, B), \sup _{b \in B} d(b, A)\right\}
$$

for all $A, B \in P_{f}(X)$.

It is well known that $\left(P_{f}(X), h\right)$ is a complete metric space and $P_{f c}(X)$ is a closed subset of it. When $Z$ is a Hausdorff topological space, a multifunction $G: Z \rightarrow P_{f}(X)$ is said to be $h$-continuous if it is continuous as a function from $Z$ into $\left(P_{f}(X), h\right)$.

Let $Y, Z$ be Hausdorff topological spaces and $G: Y \rightarrow 2^{Z} \backslash\{\phi\}$. We say that $G(\cdot)$ is 'upper semicontinuous (USC)' (resp., 'lower semicontinuous (LSC)') if for all $C \subseteq Z$ nonempty closed, $G^{-}(C)=\{y \in Y: G(y) \cap C \neq \phi\}$ (resp., $G^{+}(C)=\{y \in Y: G(y) \subseteq C\}$ ) is closed in $Y$. A USC multifunction has a closed graph in $Y \times Z$, while the converse is true if $G$ is locally compact (i.e., for every $y \in Y$, there exists a neighborhood $U$ of $y$ such that $\overline{F(U)}$ is compact in $Z$ ). A multifunction which is both USC and LSC is said to be 'continuous'. If $Y, Z$ are both metric spaces, then the above definition of LSC is equivalent to saying that for all $z \in Z$, $y \rightarrow d_{Z}(z, G(y))=\inf \left\{d_{Z}(z, v): v \in G(y)\right\}$ is upper semicontinuous as $R_{+}$-valued function. Also, lower semicontinuity is equivalent to saying that if $y_{n} \rightarrow y$ in $Y$ as $n \rightarrow \infty$, then

$$
\begin{aligned}
G(y) \subseteq \underline{\lim } G\left(y_{n}\right) & =\left\{z \in Z: \lim d_{Z}\left(z, G\left(y_{n}\right)\right)=0\right\} \\
& =\left\{z \in Z: z=\lim z_{n}, z_{n} \in G\left(y_{n}\right), n \geq 1\right\} .
\end{aligned}
$$

Let $I=[0, T]$. By $L_{1}(I, X)_{w}$, we denote the Lebesgue-Bochner space $L_{1}(I, X)$ equipped with the norm $\|g\|_{w}=\sup \left\{\left\|\int_{t}^{t^{\prime}} g(s) d s\right\|: 0 \leq t \leq t^{\prime} \leq T\right\}, g \in L_{1}(I, X)$. A set $D \subseteq L_{p}(I, X)$ is said to be 'decomposable' if for every $g_{1}, g_{2} \in D$ and for every $J \subseteq I$ measurable, we have $\chi_{J} g_{1}+\chi_{J c} g_{2} \in D$.

Let $H$ be a real separable Hilbert space, $V$ be a dense subspace of $H$ having structure of a reflexive Banach space, with the continuous embedding $V \rightarrow H \rightarrow V^{*}$, where $V^{\prime \prime}$ is the topological dual space of $V$. The system model considered here is based on this evolution triple. Let the embedding be compact. Let $\langle\cdot, \cdot\rangle$ denote the pairing of an element $x \in V^{*}$ and an element $y \in V$. If $x, y \in H$, then $\langle\cdot, \cdot\rangle=(\cdot, \cdot)$, where $(\cdot, \cdot)$ is the inner product on $H$. The norm in any Banach space $X$ will be denoted by $\|\cdot\|_{X}$. Let $1<q \leq p<\infty$ be such that $\frac{1}{p}+\frac{1}{q}=1$. We denote $L_{p}(I, V)$ by $X$. Then the dual space of $X$ is $L_{q}\left(I, V^{*}\right)$ and is denoted by $X^{*}$. For $p, q$ satisfying the above conditions, from reflexivity of $V$ that both $X$ and $X^{*}$ are reflexive Banach spaces (see Zeidler [23, p.411]).

Define $W_{p q}(I)=\left\{x: x \in X, \dot{x} \in X^{*}\right\}$, where the derivative in this definition should be understood in the sense of distribution. Furnished with the norm $\|x\|_{W_{p q}}=\|x\|_{X}+\|\dot{x}\|_{X^{n}}$, the space $\left(W_{p q}(I),\|x\|_{W_{p q}}\right)$ becomes a Banach space which is clearly reflexive and separable. Moreover, $W_{p q}(I)$ embeds into $C(I, H)$ continuously (see Proposition 23.23 of [23]). So, every element in $W_{p q}(I)$ has a representative in $C(I, H)$. Since the embedding $V \rightarrow H$ is compact, the embedding $W_{p q}(I) \rightarrow L_{p}(I, H)$ is also compact (see Problem 23.13 of [23]). 
The pairing between $X$ and $X^{*}$ is denoted by $\left\langle\langle\cdot, \cdot\rangle\right.$. By ' ${ }^{\prime}$ ' we denote the weakly convergence. The following lemmas are still needed in the proof of our main theorems.

Lemma 2.1 (see [24]) If $X$ is a Banach space, $C \subset X$ is nonempty, closed and convex with $0 \in C$ and $G: C \rightarrow P_{k c}(C)$ is an upper semicontinuous multifunction which maps bounded sets into relatively compact sets, then one of the following statements are true:

(i) the set $\Gamma=\{x \in C: x \in \lambda G(x), \lambda \in(0,1)\}$ is unbounded;

(ii) the $G(\cdot)$ has a fixed point, i.e., there exists $x \in C$ such that $x \in G(x)$.

Let $X$ be a Banach space and let $L^{2}(I, X)$ be the Banach space of all functions $u: I \rightarrow X$ which are Bochner integrable. $D\left(L^{2}(I, X)\right)$ denotes the collection of nonempty decomposable subsets of $L^{2}(I, X)$. Now, let us state the Bressan-Colombo continuous selection theorem.

Lemma 2.2 (see [25]) Let $X$ be a separable metric space and let $F: X \rightarrow D\left(L^{2}(I, X)\right)$ be a lower semicontinuous multifunction with closed decomposable values. Then $F$ has a continuous selection.

Let $X$ be a separable Banach space and $C(I, X)$ be the Banach space of all continuous functions. A multifunction $F: I \times X \rightarrow P_{w k c}(X)$ is said to be Carathéodory type if for every $x \in X, F(\cdot, x)$ is measurable, and for almost all $t \in I, F(t, \cdot)$ is $h$-continuous (i.e., it is continuous from $X$ to the metric space $\left(P_{f}(X), h\right)$, where $h$ is a Hausdorff metric).

Let $M \subset C(I, X)$, a multifunction $F: I \times X \rightarrow P_{w k c}(X)$ is called integrably bounded on $M$ if there exists a function $\lambda: I \rightarrow R_{+}$such that for almost all $t \in I$, $\sup \{\|y\|: y \in$ $F(t, x(t)), x(\cdot) \in M\} \leq \lambda(t)$. A nonempty subset $M_{0} \subset C(I, X)$ is called $\sigma$-compact if there is a sequence $\left\{M_{k}\right\}_{k \geq 1}$ of compact subsets $M_{k}$ such that $M_{0}=\bigcup_{k \geq 1} M_{k}$. Let $M_{0} \subset M$ be such that $M_{0}$ is dense in $M$ and $\sigma$-compact. The following continuous selection theorem in the extreme point case is due to Tolstonogov [26].

Lemma 2.3 (see [26]) Let the multifunction $F: I \times X \rightarrow P_{w k c}(X)$ be Carathéodory type and integrably bounded. Then there exists a continuous function $g: M \rightarrow L_{p}(I, X)$ such that for almost all $t \in I$, if $x(\cdot) \in M_{0}$, then $g(x)(t) \in \operatorname{ext} F(t, x(t))$, and if $x(\cdot) \in M \backslash M_{0}$, then $g(x)(t) \in \overline{\operatorname{ext}} F(t, x(t))$.

\section{Main results}

Let $I=[0, T]$, consider the following evolution inclusions:

$$
\begin{aligned}
& \dot{x}+A(t, x)+B x \in F(t, x), \quad \text { a.e. } I, \\
& x(0)=\varphi(x),
\end{aligned}
$$

where $A: I \times V \rightarrow V^{\prime \prime}$ is a nonlinear map, $B: V \rightarrow V^{*}$ is a bounded linear map, $\varphi: H \rightarrow H$ is a continuous map and $F: I \times H \rightarrow 2^{V^{*}}$ is a multifunction satisfying some conditions mentioned later.

Definition 3.1 A function $x \in W_{p q}(I)$ is called a solution to the problem (3.1) iff

$$
\langle\dot{x}(t), v\rangle+\langle A(t, x(t)), v\rangle+\langle B x(t), v\rangle=\langle f(t), v\rangle,
$$

where $x(0)=\varphi(x), f(t) \in F(t, x(t))$ for all $v \in V$ and almost all $t \in I$. 
We will need the following hypotheses on the data problem (3.1).

(H1) $A: I \times V \rightarrow V^{*}$ is an operator such that

(i) $t \rightarrow A(t, x)$ is measurable;

(ii) for each $t \in I$, the operator $A(t, \cdot): V \rightarrow V^{*}$ is uniformly monotone and hemicontinuous, that is, there exists a constant $C_{1}>0$ (independent of $t$ ) such that

$$
\left\langle A\left(t, x_{1}\right)-A\left(t, x_{2}\right), x_{1}-x_{2}\right\rangle \geq C_{1}\left\|x_{1}-x_{2}\right\|_{H}^{p}
$$

for all $x_{1}, x_{2} \in V$, and the map $s \mapsto\langle A(t, x+s z), y\rangle$ is continuous on $[0,1]$ for all $x, y, z \in V$;

(iii) there exist a constant $C_{2}>0$, a nonnegative function $a(\cdot) \in L_{q}(I)$ and a nondecreasing continuous function $\eta(\cdot) \in L_{q}(I)$ such that $\|A(t, x)\|_{V^{*}} \leq a(t)+C_{2} \eta\left(\|x\|_{V}\right)$ for all $x \in V$, a.e. on $I$;

(iv) there exist $C_{3}>0, C_{4}>0, b(\cdot) \in L_{1}(I)$ such that

$$
\langle A(t, x), x\rangle \geq C_{3}\|x\|_{V}^{p}-C_{4}\|x\|_{V}^{p-1}+\frac{1}{2 T}\|x(0)\|^{2}-b(t) \quad \text { a.e. } I, \forall x \in V,
$$

or

$$
\langle A(t, x), x\rangle \geq C_{3}\|x\|_{V}^{p}-C_{4}\|x\|_{V}^{p-1}-b(t) \quad \text { a.e. } I, \forall x \in V, p>2 .
$$

(H2) $F: I \times H \rightarrow P_{k}\left(V^{*}\right)$ is a multifunction such that

(i) $(t, x) \rightarrow F(t, x)$ is graph measurable;

(ii) for almost all $t \in I, x \rightarrow F(t, x)$ is LSC;

(iii) there exist a nonnegative function $b_{1}(\cdot) \in L_{q}(I)$ and a constant $C_{5}>0$ such that

$$
|F(t, x)|=\sup \left\{\|f\|_{V^{\circ}}: f \in F(t, x)\right\} \leq b_{1}(t)+C_{5}\|x\|_{H}^{k-1} \quad \forall x \in V \text { a.e. } I
$$

where $1 \leq k<p$.

(H3) (i) $B: V \rightarrow V^{*}$ is a bounded linear self-adjoint operator such that $(B x, x) \geq 0$ for all $x \in V$, a.e. on $I$;

(ii) there exists a continuous function $\varphi: L_{p}(I, H) \rightarrow H$ such that

$$
\|\varphi(u)-\varphi(v)\| \leq\|u-v\|_{C(I, H)} \quad \forall u, v \in C(I, H)
$$

and $\varphi(0)=0$.

It is convenient to rewrite the system (3.1) as an operator equation in $W_{p q}(I)$. For $x \in X$, we get

$$
\begin{array}{ll}
A(x)(t)=A(t, x), & B(x)(t)=B x(t), \\
F(x)(t)=F(t, x(t)), & t \in I .
\end{array}
$$

It follows from Theorem 30.A of Zeidler [23] that the operator $A: X \rightarrow X^{*}$ is bounded, monotone, hemicontinuous and coercive. By using the same technique, one can show that 
the operator $F: L_{p}(I, H) \rightarrow X^{*}$ is bounded and satisfies

$$
|F(t, x)|=\sup \left\{\|f\|_{X^{*}}: f \in F(t, x)\right\} \leq \hat{M}_{1}+\hat{M}_{2}\|x\|_{L_{p}(I, H)}^{k-1}
$$

for some constants $\hat{M}_{1}, \hat{M}_{2}>0$ and all $x \in L_{p}(I, H)$.

We define

$$
L u=\dot{u}, \quad D(L)=\left\{u \in W_{p q}(I): u(0)=\xi \in H\right\},
$$

where $\dot{u}$ stands for the generalized derivative of $u$, i.e.,

$$
\int_{0}^{T} \dot{u}(t) v(t) d t=-\int_{0}^{T} u(t) \dot{v}(t) d t \quad \forall v(\cdot) \in C_{0}^{\infty}(I)
$$

For the proofs of main results, we need the following lemma.

Lemma 3.1 Let $V \subseteq H \subseteq V^{*}$ be an evolution triple and let $X=L_{p}(I, V)$, where $1<p<\infty$ and $0<T<\infty$. Then the linear operator $L: D(L) \subseteq X \rightarrow X^{*}$ defined by (3.2) is maximal monotone.

Proof In the sequel we will show that $L$ is maximal monotone. To prove this, suppose that $(v, w) \in X \times X^{*}$ and

$$
0 \leq\langle w-L u, v-u\rangle \quad \forall u \in D(L)
$$

We have to show that $v \in D(L)$ and $w=L v$, i.e., $w=\dot{v}$. Due to the arbitrariness of $u$, we choose $u=\phi z+\xi$, where $\phi \in C_{0}^{\infty}(I), \xi=u_{0}$ and $z \in V$. Then $\dot{u}=\dot{\phi} z$, so $\langle L u, u\rangle=0$. From $\langle w-L u, v-u\rangle \geq 0$, we obtain that

$$
0 \leq\left\langle\langle w, v-\xi\rangle-\int_{0}^{T}\langle\dot{\varphi} v+\varphi w, z\rangle d t \quad \forall z \in V .\right.
$$

By the arbitrariness of $z$, one has that

$$
\int_{0}^{T}(\dot{\varphi} v+\varphi w) d t=0 \quad \forall \varphi \in C_{0}^{\infty}(I)
$$

Hence, $w=\dot{v}$. Since $v \in W_{p q}(I)$, then $w \in X^{*}$. It remains to show that $v \in D(L)$. Using the integration by parts formula for functions in $W_{p q}(I)$ (see Zeidler [23], Proposition 23.23), we obtain from (3.2) that

$$
0 \leq\langle\dot{v}-\dot{u}, v-u\rangle=\frac{1}{2}\left(\|v(T)-u(T)\|^{2}-\|v(0)-u(0)\|^{2}\right) \quad \forall u \in D(L) .
$$

Choose a set of functions $\left(a_{n}\right)_{n \geq 1}$ in $H$ such that $T a_{n} \rightarrow v(T)-\xi$ as $n \rightarrow \infty$. For $\xi \in H$, let $u(t)=t a_{n}+\xi$, then $u \in D(L)$. By (3.3), we have $v(0)=u(0)=\xi$ as $n \rightarrow \infty$. Hence, $v \in D(L)$. This completes the proof.

Theorem 3.1 If hypotheses (H1), (H2) and (H3) hold, the problem (3.1) has at least one solution. 
Proof The process of proof is divided into four parts.

Step 1. We claim that the equation

$$
\begin{aligned}
& \dot{x}+A(t, x)+B x=f(t) \quad \text { a.e. } I, \\
& x(0)=(1-\epsilon) \varphi(x)
\end{aligned}
$$

has only one solution.

Firstly, for every $\epsilon \in(0,1], y \in X$ and $f \in X^{\prime \prime}$, we claim that the equation

$$
\begin{aligned}
& \dot{x}+A(t, x)+B x=f(t) \quad \text { a.e. } I, \\
& x(0)=(1-\epsilon) \varphi(y)
\end{aligned}
$$

has only one solution. By $(\mathrm{H} 1)$ and $(\mathrm{H} 3)$, it is easy to check that $(A+B)$ is bounded, monotone, hemicontinuous and coercive. Moreover, by Lemma 3.1, $L$ is a linear maximal monotone operator. Therefore, $R(L+A+B)=V^{*}$, i.e., $L+(A+B)$ is surjective (see [23, p.868]). The uniqueness is clear. Hence, for the Cauchy problem (3.5) has a unique $x_{y}(t) \in W_{p q}(I)$. By $W_{p q}(I) \subset C(I, H)$, then the operator $P: W_{p q}(I) \rightarrow W_{p q}(I)$ is defined as follows:

$$
P(y)=\int_{0}^{t} \dot{x}(s) d s+(1-\epsilon) \varphi(y) .
$$

By (3.5), we have

$$
P\left(y_{1}\right)-P\left(y_{2}\right)+\int_{0}^{t} A\left(s, x_{1}\right)-A\left(s, x_{2}\right) d s=(1-\epsilon) \varphi\left(y_{2}\right)-(1-\epsilon) \varphi\left(y_{1}\right)
$$

for all $y_{1}, y_{2} \in W_{p q}(I)$. Take an inner product over (3.5) with $x_{1}-x_{2}$, then

$$
\left(P\left(y_{1}\right)-P\left(y_{2}\right), x_{1}-x_{2}\right)+\int_{0}^{t}\left(A\left(s, x_{1}\right)-A\left(s, x_{2}\right), x_{1}-x_{2}\right) d s=(1-\epsilon)\left(\varphi\left(y_{2}\right)-\varphi\left(y_{1}\right), x_{1}-x_{2}\right) .
$$

By (H1)(ii), we have

$$
\left\|P\left(y_{1}\right)-P\left(y_{2}\right)\right\|^{2} \leq(1-\epsilon)\left\|\varphi\left(y_{2}\right)-\varphi\left(y_{1}\right)\right\|\left\|x_{1}-x_{2}\right\| .
$$

Hence,

$$
\begin{aligned}
\left\|P\left(y_{1}\right)-P\left(y_{2}\right)\right\|_{C(I, H)} & \leq(1-\epsilon)\left\|\varphi\left(y_{2}\right)-\varphi\left(y_{1}\right)\right\|_{C(I, H)} \\
& \leq(1-\epsilon)\left\|y_{2}-y_{1}\right\|_{C(I, H)} .
\end{aligned}
$$

Invoking the Banach fixed point theorem, the operator $P$ has only one fixed point $x_{\epsilon}=$ $P\left(x_{\epsilon}\right)$, i.e., $x_{\epsilon}$ is the uniform solution of (3.4).

Therefore, we define $L_{\epsilon}: W_{p q}(I) \rightarrow X^{*}$ as $L_{\epsilon} x=\dot{x}+A(t, x)+B x$ and $x(0)=(1-\epsilon) \varphi(x)$. By Step 1, we have $L_{\epsilon}: W_{p q}(I) \rightarrow X^{*}$ is one-to-one and surjective, and so $L_{\epsilon}^{-1}: X^{*} \rightarrow W_{p q}(I)$ is well defined.

Step 2. $L_{\epsilon}^{-1}: X^{*} \rightarrow L_{p}(I, H)$ is completely continuous. 
We only need to show that $L_{\epsilon}^{-1}$ is continuous and maps a bounded set into a relatively compact set. We claim that $L_{\epsilon}: W_{p q}(I) \rightarrow X^{*}$ is continuous. In fact, let $\left\{x_{n}\right\}_{n \geq 1} \subset W_{p q}(I)$ such that $x_{n} \rightarrow x$ as $n \rightarrow \infty$. From (H1)(ii) and (H3), we infer that $x_{n} \rightarrow x, A\left(x_{n}\right) \rightarrow A(x)$, $B x_{n} \rightarrow B x$, a.e. $I$ as $n \rightarrow \infty$. Obviously, $\varphi\left(x_{n}\right) \rightarrow \varphi(x)$. Therefore, $L_{\epsilon}: W_{p q}(I) \rightarrow X^{*}$ is continuous and $L_{\epsilon}^{-1}$ is continuous.

Let $K \subset X^{*}$ be a bound set, for any $f \in K$, there is a priori bound in $W_{p q}(I)$ for the possible solution $x(t)=L_{\epsilon}^{-1} f$ of (3.4). Then

$$
\dot{x}+A(t, x)+B x=f(t) \quad \text { a.e. } I .
$$

It follows that

$$
\langle\langle\dot{x}, x\rangle\rangle+\langle\langle A x, x\rangle\rangle+\langle\langle B x, x\rangle\rangle=\langle\langle f(t), x\rangle\rangle
$$

By (H1)(iv),

$$
\langle A u, u\rangle \geq C_{3}\|u\|_{X}^{p}-C_{4}\|u\|_{X}^{p-1}+\frac{1}{2}\|u(0)\|^{2}-\|b\|_{L^{1}},
$$

or

$$
\langle A u, u\rangle \geq C_{3}\|u\|_{X}^{p}-C_{4}\|u\|_{X}^{p-1}-\|b\|_{L^{1}}
$$

with $p>2$. But

$$
\begin{aligned}
& \langle\dot{u}, u\rangle\rangle=\|u(T)\|^{2}-\|u(0)\|^{2}, \\
& \langle f, u\rangle \leq\|f\|_{X^{*}}\|u\|_{X} .
\end{aligned}
$$

Therefore,

$$
C_{3}\|x\|_{X}^{p} \leq C_{4}\|x\|_{X}^{p-1}+\|f\|_{X^{*}}\|x\|_{X}+\|b\|_{L^{1}}
$$

or

$$
C_{3}\|x\|_{X}^{p} \leq C_{4}\|x\|_{X}^{p-1}+\|f\|_{X^{n}}\|x\|_{X}+\|b\|_{L^{1}}+\|x\|_{X}^{2},
$$

with $p>2$. So, there exists an $M_{1}>0$ such that $\|x\|_{X} \leq M_{1}$. Because of the boundedness of operators $A, B$, we obtain that there exists an $M_{2}>0$ such that $\|\dot{x}\|_{X^{n}} \leq M_{2}$. Hence, $\|x\|_{W_{p q}} \leq M$ for some constant $M>0$. Therefore, we have $L_{\epsilon}^{-1}(K)$ is bounded in $W_{p q}(I)$. But $W_{p q}(I)$ is compactly embedded in $L_{p}(I, H)$. Therefore, $L_{\epsilon}^{-1}(K)$ is relatively compact in $L_{p}(I, H)$.

Let $\widehat{N}: L_{p}(I, H) \rightarrow 2^{X^{\circ}}$ be a multivalued Nemitsky operator corresponding to $F$ and $\widehat{N}$ was defined by $\widehat{N}(x)=\left\{v \in X^{*}: v(t) \in F(t, x(t))\right\}$ a.e. on $I$.

Step 3. $\widehat{N}(\cdot)$ has nonempty, closed, decomposable values and is LSC.

The closedness and decomposability of the values of $\widehat{N}(\cdot)$ are easy to check. For the nonemptiness, note that if $x \in L_{p}(I, H)$, by the hypothesis (H2)(i), $(t, x) \rightarrow F(t, x)$ is graph measurable, so we apply Aumann's selection theorem and obtain a measurable map $v$ : 
$I \rightarrow V^{*}$ such that $v(t) \in F(t, x(t))$ a.e. on $I$. By the hypothesis (H2)(iii), $v \in X^{*}$. Thus, for every $x \in L_{p}(I, H), \widehat{N}(x) \neq \emptyset$. To prove the lower semicontinuity of $\widehat{N}(\cdot)$, we only need to show that every $u \in X^{\prime \prime}, x \rightarrow d(u, \widehat{N}(x))$ is a USC $R_{+}$-valued function. Note that

$$
\begin{aligned}
d(u, \widehat{N}(x)) & =\inf \left\{\|u-v\|_{X^{*}}: v \in \widehat{N}(x)\right\} \\
& =\inf \left\{\left[\int_{0}^{T}\|u(t)-v(t)\|_{V^{*}}^{q} d t\right]^{1 / q}: v \in \widehat{N}(x)\right\} \\
& =\left\{\int_{0}^{T} \inf \left\{\|u(t)-v(t)\|_{V^{*}}^{q}: v \in \widehat{N}(x)\right\} d t\right\}^{1 / q} \\
& =\left\{\int_{0}^{T}[d(u(t), F(t, x(t)))]^{q} d t\right\}^{1 / q}
\end{aligned}
$$

(see Hiai and Umegaki [27] Theorem 2.2). We will show that for every $\lambda \geq 0$, the superlevel set $U_{\lambda}=\left\{x \in L_{p}(I, H): d(u, \widehat{N}(x)) \geq \lambda\right\}$ is closed in $L_{p}(I, H)$. Let $\left\{x_{n}\right\}_{n \geq 1} \subseteq U_{\lambda}$ and assume that $x_{n} \rightarrow x$ in $L_{p}(I, H)$. By passing to a subsequence if necessary, we may assume that $x_{n}(t) \rightarrow x(t)$ a.e. on $I$ as $n \rightarrow \infty$. By (H2)(ii), $x \rightarrow d(u(t), F(t, x))$ is an upper semicontinuous $R_{+}$-valued function. So, via Fatou's lemma, we have

$$
\begin{aligned}
\lambda^{q} & \leq \varlimsup \\
& =\varlimsup \int_{0}^{T}\left[d\left(u, \widehat{N}\left(x_{n}\right)\right)\right]^{q} \\
& \left.\left.\leq \int_{0}^{T} \varlimsup(t), F\left(t, x_{n}(t)\right)\right)\right]^{q} d t \\
& \leq \int_{0}^{T}\left[d\left(u(t), F\left(t, x_{n}(t)\right)\right)\right]^{q} d t
\end{aligned}
$$

Therefore, $x \in U_{\lambda}$ and this proves the LSC of $\widehat{N}(\cdot)$. By Lemma 2.2, we obtain a continuous $\operatorname{map} f: L_{p}(I, H) \rightarrow X^{\prime \prime}$ such that $f(x) \in \widehat{N}(x)$. To finish our proof, we need to solve the fixed point problem: $x=L_{\epsilon}^{-1} \circ f(x)$.

Since the embedding $V \rightarrow H$ is compact, the embedding $W_{p q}(I) \rightarrow L_{p}(I, H)$ is compact. That is, $x_{n} \rightarrow x$ in $L_{p}(I, H)$ whenever $x_{n} \rightarrow x$ in $W_{p q}(I)$. By using the above relation and the continuity of $f$, we have $f\left(x_{n}\right) \rightarrow f(x)$ in $X^{*}$ whenever $x_{n} \rightarrow x$ in $W_{p q}(I)$. So, $L_{\epsilon}^{-1} \circ f$ : $L_{p}(I, H) \rightarrow L_{p}(I, H)$ is compact.

Step 4. We claim that the set $\Gamma=\left\{x \in L_{p}(I, H): x=\sigma L_{\epsilon}^{-1} \circ f(x), \sigma \in(0,1)\right\}$ is bounded.

Let $x \in \Gamma$, then we have $L_{\epsilon}\left(\frac{x}{\sigma}\right)=f(x)$. Note that

$$
\|\left\langle\frac{\dot{x}}{\sigma}, \frac{x}{\sigma}\right\rangle+\left\langle\left\langle A\left(\frac{x}{\sigma}\right), \frac{x}{\sigma}\right\rangle\right\rangle+\left\langle\left\langle B \frac{x}{\sigma}, \frac{x}{\sigma}\right\rangle=\left\langle\left\langle f(x), \frac{x}{\sigma} \| .\right.\right.\right.
$$

By (H1)(iv) and (H3)(i), one has that

$$
\langle\langle A u, u\rangle\rangle+\langle\langle B x, x\rangle\rangle \geq C_{3}\|u\|_{X}^{p}-C_{4}\|u\|_{X}^{p-1}+\frac{1}{2}\|u(0)\|^{2}-\|b\|_{L^{1}},
$$

or

$$
\left\langle\langle A u, u\rangle+\langle\langle B x, x\rangle\rangle \geq C_{3}\|u\|_{X}^{p}-C_{4}\|u\|_{X}^{p-1}-\|b\|_{L^{1}}\right.
$$


with $p>2$. By using the integration by parts formula, we have

$$
\begin{aligned}
\| & \left.A\left(\frac{x}{\sigma}\right), \frac{x}{\sigma}\right\rangle+\left\langle\left\langle B \frac{x}{\sigma}, \frac{x}{\sigma}\right\rangle\right) \\
& =\left\langle\left\langle f(x), \frac{x}{\sigma}\right\rangle\right\rangle-\left\langle\left\langle\frac{\dot{x}}{\sigma}, \frac{x}{\sigma}\right\rangle\right) \\
& \leq \frac{1}{\sigma}\left\{\int_{0}^{T}\|f(t, x)\|_{V^{*}}^{q} d t\right\}^{1 / q}\left\{\int_{0}^{T}\|x\|_{V}^{p} d t\right\}^{1 / p}+\frac{1}{2 \sigma^{2}}\|\varphi(x)\|^{2} \\
& \leq \frac{1}{\sigma}\left\{\int_{0}^{T}\left(h(t)+\|x\|_{H}^{k-1}\right)^{q} d t\right\}^{1 / q}\|x\|_{X}+\frac{1}{2 \sigma^{2}}\|\varphi(x)\|^{2} \\
& \leq \frac{2}{\sigma}\left\{\int_{0}^{T}|h(t)|^{q}+\|x\|_{H}^{q(k-1)} d t\right\}^{1 / q}\|x\|_{X}+\frac{1}{2 \sigma^{2}}\|\varphi(x)\|^{2} \\
& \leq \frac{2}{\sigma}\left\{\left(\int_{0}^{T}|h(t)|^{q}\right)^{1 / q}+\left(\int_{0}^{T}\|x\|_{H}^{q(k-1)} d t\right)^{1 / q}\right\}\|x\|_{X}+\frac{1}{2 \sigma^{2}}\|\varphi(x)\|^{2} \\
& \leq \gamma_{1}\|x\|_{X}+\gamma_{2}\|x\|_{X}^{k}+\frac{1}{2 \sigma^{2}}\|\varphi(x)\|^{2},
\end{aligned}
$$

where $\gamma_{1}, \gamma_{2}>0$. By (3.9), (3.10) and (3.11), if $1 \leq k<p$, then we have

$$
\|x\|_{X}^{p} \leq \beta_{1}\|x\|_{X}^{p-1}+\beta_{2}\|x\|_{X}^{k}+\beta_{3}\|x\|_{X}+\beta_{4}\|b\|_{L^{1}} .
$$

If $1 \leq k<p, p>2$, then we have

$$
\|x\|_{X}^{p} \leq \beta_{1}\|x\|_{X}^{p-1}+\beta_{2}\|x\|_{X}^{k}+\beta_{3}\|x\|_{X}+\beta_{4}\|b\|_{L^{1}}+\beta_{5}\|x\|_{X}^{2} .
$$

Thus, by virtue of the inequalities (3.12) and (3.13), we can find a constant $M_{1}>0$ such that $\|x\|_{X} \leq M_{1}$ for all $x \in \Gamma$. From the boundedness of operators $A, B$ and $f$, and the continuous embedding $X \rightarrow L_{p}(I, H)$, we obtain $\|A(x)\|_{X^{*}} \leq M_{2},\|B x\|_{X^{*}} \leq M_{3}$ and $\|f(x)\|_{X^{*}} \leq M_{4}$ for some constants $M_{2}>0, M_{3}>0, M_{4}>0$ and all $x \in \Gamma$. Therefore,

$$
\|\dot{x}\|_{X^{*}} \leq\|A(x)\|_{X^{*}}+\|B x\|_{X^{*}}+\|f(x)\|_{X^{*}} \leq M_{2}+M_{3}+M_{4}
$$

for all $x \in \Gamma$.

It follows from (3.14) that $\|x\|_{W_{p q}} \leq\|x\|_{X}+\|\dot{x}\|_{X^{*}} \leq \hat{M}$ for some constant $\hat{M}>0$. Hence, $\Gamma$ is a bounded subset of $W_{p q}(I)$. So, $\Gamma$ is a bounded subset of $L_{p}(I, H)$ since the embedding $W_{p q}(I) \rightarrow L_{p}(I, H)$ is compact.

Invoking the Leray-Schauder theorem, one has that there exists an $x_{\epsilon} \in W_{p q}(I)$ such that $x_{\epsilon}=L_{\epsilon}^{-1} f\left(x_{\epsilon}\right)$, i.e., $x_{\epsilon}$ is a solution of the following problem:

$$
\begin{aligned}
& \dot{x}_{\epsilon}+A\left(t, x_{\epsilon}\right)+B x_{\epsilon}=f\left(x_{\epsilon}\right), \\
& f\left(x_{\epsilon}\right) \in F\left(t, x_{\epsilon}\right) \quad \text { a.e. } I, \\
& x_{\epsilon}(0)=(1-\epsilon) \varphi\left(x_{\epsilon}\right) .
\end{aligned}
$$


Let $\left(\epsilon_{n}\right)_{n \geq 1} \subset(0,1)$ and $\epsilon_{n} \rightarrow 0$. For every $n \in N$, there exists an $x_{n} \in W_{p q}(I)$ which is a solution of the following equations:

$$
\begin{aligned}
& \dot{x}_{n}+A\left(t, x_{n}\right)+B x_{n}=f\left(x_{n}\right) \text { a.e. } I, \\
& f\left(x_{n}\right) \in F\left(t, x_{n}\right), \\
& x_{n}(0)=\left(1-\epsilon_{n}\right) \varphi\left(x_{n}\right) .
\end{aligned}
$$

By Step 3, we have that $\left\{x_{n}\right\}_{n \geq 1}$ is uniformly bounded. By the boundedness of the sequence $\left\{x_{n}\right\}_{n \geq 1} \subset W_{p q}(I)$, it follows that the sequence $\left\{\dot{x}_{n}\right\}_{n \geq 1} \subset X^{*}$ is uniformly bounded and passing to subsequence if necessary, we may assume that $\dot{x}_{n} \rightarrow u$ in $X^{*}$. Evidently, $u=\dot{x}$ and $x_{n} \rightarrow x$ in $W_{p q}(I)$. Since the embedding $W_{p q}(I) \hookrightarrow L_{p}(I, H)$ is compact, then $x_{n} \rightarrow x$ in $L_{p}(I, H)$. Hence, from the hypothesis (H2)(ii), we obtain $f\left(x_{n}\right) \rightarrow f(x)$ and $f(x) \in F(t, x)$. Since the operator $A$ is hemicontinuous and monotone and $B$ is a continuous linear operator, thus $A\left(x_{n}\right) \rightarrow A(x), B x_{n} \rightarrow B x$ in $X^{*}$ as $n \rightarrow \infty$. Therefore, we obtain $\dot{x}+A(x)+B x=f$, $f \in F(t, x)$ a.e. on $I$. Since $x_{n}(t) \rightarrow x(t)$ in $L_{p}(I, H)$ and $\varphi: L_{p}(I, H) \rightarrow H$ is continuous, then we have

$$
x_{n}(0)=\left(1-\epsilon_{n}\right) \varphi\left(x_{n}\right) \rightarrow \varphi(x)=x(0) .
$$

Hence, $x$ is a solution of (3.1). The proof is completed.

Next, we consider the convex case, the assumption on $F$ is as follows:

(H4) $F: I \times H \rightarrow P_{k c}\left(V^{*}\right)$ is a multifunction such that

(i) $(t, x) \rightarrow F(t, x)$ is graph measurable;

(ii) for almost all $t \in I, x \rightarrow F(t, x)$ has a closed graph; and (H2)(iii) hold.

Theorem 3.2 If hypotheses (H1), (H3) and (H4) hold, the problem (3.1) has at least one solution; moreover, the solution set is weakly compact in $W_{p q}(I)$.

Proof The proof is as that of Theorem 3.1. So, we only present those particular points where the two proofs differ.

In this case, the multivalued Nemistsky operator $\widehat{N}: L_{p}(I, H) \rightarrow 2^{X_{w}^{*}}$ has nonempty closed, convex values in $X^{*}$ and is USC from $L_{p}(I, H)$ into $X^{*}$ furnished with the weak topology (denoted by $X_{w}^{*}$ ). The closedness and convexity of the values of $\widehat{N}(\cdot)$ are clear. To prove the nonemptiness, let $x \in L_{p}(I, H)$ and $\left\{s_{n}\right\}_{n \geq 1}$ be a sequence of step functions such that $s_{n}(t) \rightarrow x(t)$ in $H$ and $\left\|s_{n}(t)\right\|_{H} \leq\|x(t)\|_{H}$ a.e. on $I$. Then by virtue of the hypothesis (H4)(i), for every $n \geq 1, t \rightarrow F\left(t, s_{n}(t)\right)$ admits a measurable selector $v_{n}(t)$. From the hypothesis (H4)(iii), we have that $\left\|v_{n}\right\|_{X^{*}} \leq \hat{M}_{1}+\hat{M}_{2}\|x\|_{L_{p}(I, H)}^{k-1}$, so $\left\{v_{n}(t)\right\}_{n \geq 1} \subseteq X^{* \prime}$ is uniformly integrable. So, by the Dunford-Pettis theorem, and by passing to a subsequence if necessary, we may assume that $v_{n} \rightarrow v$ weakly in $X^{*}$. Then from Theorem 3.1 in [28], we have

$$
v(t) \in \overline{\operatorname{conv}} \varlimsup\left\{v_{n}(t)\right\}_{n \geq 1} \subseteq \overline{\operatorname{conv}} \varlimsup F\left(t, s_{n}(t)\right) \subseteq F(t, x(t)) \quad \text { a.e. on } I,
$$

the last inclusion being a consequence of the hypothesis (H4)(ii). So, $v \in \widehat{N}(x)$, which means that $\widehat{N}(\cdot)$ is nonempty. 
Next, we show that $\widehat{N}(\cdot)$ is USC from $L_{p}(I, H)$ into $X_{w}^{*}$. Let $\Xi$ be a nonempty and weakly closed subset of $X^{*}$. Obviously, it is sufficient to show that the set

$$
\widehat{N}^{-1}(\Xi)=\left\{x \in L_{p}(I, H): \widehat{N}(x) \cap \Xi \neq \phi\right\}
$$

is closed. Let $\left\{x_{n}\right\}_{n \geq 1} \subseteq \widehat{N}^{-1}(\Xi)$ and assume $x_{n} \rightarrow x$ in $L_{p}(I, H)$. Passing to a subsequence, we can get that $x_{n}(t) \rightarrow x(t)$ a.e. on $I$. Let $f_{n} \in \widehat{N}\left(x_{n}\right) \cap \Xi, n \geq 1$. Then by virtue of the hypothesis (H4)(iii), we have

$$
\left\|f_{n}\right\|_{X^{n}} \leq \hat{M}_{1}+\hat{M}_{2}\|x\|_{L_{p}(I, H)}^{k-1}
$$

So, by the Dunford-Pettis theorem, we may assume that $f_{n} \rightarrow f \in \Xi$ in $X_{w}^{*}$. As before, we have

$$
f(t) \in \overline{\operatorname{conv}} \varlimsup\left\{f_{n}(t)\right\}_{n \geq 1} \subseteq \overline{\operatorname{conv}} \varlimsup \overline{\lim } F\left(t, x_{n}(t)\right) \subseteq F(t, x(t)) \quad \text { a.e. on } I,
$$

then $f \in \widehat{N}(x) \cap \Xi$, i.e., $\widehat{N}^{-1}(\Xi)$ is closed in $L_{p}(I, H)$. This proves the upper semicontinuity of $\widehat{N}(\cdot)$ from $L_{p}(I, H)$ into $X_{w}^{*}$.

We consider the following fixed point problem:

$$
x \in L_{\epsilon}^{-1} \circ \widehat{N}(x) .
$$

Recalling that $L_{\epsilon}^{-1}: X^{*} \rightarrow L_{p}(I, H)$ is completely continuous, we see that $L_{\epsilon}^{-1} \circ \widehat{N}$ : $L_{p}(I, H) \rightarrow P_{k c}\left(L_{p}(I, H)\right)$ is USC and maps bounded sets into relatively compact sets. We easily check that

$$
\Gamma_{1}=\left\{x \in L_{p}(I, H): x \in \lambda L_{\epsilon}^{-1} \circ \widehat{N}(x), \lambda \in(0,1)\right\}
$$

is bounded, as a proof of Theorem 3.1. Invoking the Leray-Schauder fixed point theorem, one has that there exists an $x_{\epsilon} \in W_{p q}(I)$ such that $x_{\epsilon} \in L_{\epsilon}^{-1} \circ \hat{N}\left(x_{\epsilon}\right)$, i.e., $x_{\epsilon}$ is a solution of the following problem:

$$
\begin{aligned}
& \dot{x}_{\epsilon}+A\left(t, x_{\epsilon}\right)+B x_{\epsilon} \in F\left(t, x_{\epsilon}\right) \text { a.e. } I, \\
& x_{\epsilon}(0)=(1-\epsilon) \varphi\left(x_{\epsilon}\right) .
\end{aligned}
$$

Let $\left(\epsilon_{n}\right)_{n \geq 1} \subset(0,1)$ and $\epsilon_{n} \rightarrow 0$. For every $n \in N$, there exists an $x_{n} \in W_{p q}(I)$ which is a solution of the following problem:

$$
\begin{aligned}
& \dot{x}_{n}+A\left(t, x_{n}\right)+B x_{n}=f_{n}(t) \quad \text { a.e. } I, \\
& f_{n}(t) \in F\left(t, x_{n}\right) \\
& x_{n}(0)=\left(1-\epsilon_{n}\right) \varphi\left(x_{n}\right) .
\end{aligned}
$$

By Step 3, $\left\{x_{n}\right\}_{n \geq 1}$ is uniformly bounded. By the boundedness of the sequence $\left\{x_{n}\right\}_{n \geq 1} \subset$ $W_{p q}(I)$, it follows that the sequence $\left\{\dot{x}_{n}\right\}_{n \geq 1} \subset X^{*}$ is uniformly bounded and, passing to subsequence if necessary, we may assume that $\dot{x}_{n} \rightarrow \dot{x}$ in $X^{*}$. Thus, $A\left(x_{n}\right) \rightarrow A(x), B x_{n} \rightarrow$ 
$B x$ in $X^{*}$ as $n \rightarrow \infty$. Evidently, there exists $f_{n} \in N\left(x_{n}\right)$, by virtue of the hypothesis (H4)(iv), we have that $\left\|f_{n}\right\|_{X^{*}} \leq \hat{M}_{1}+\hat{M}_{2}\|x\|_{L_{p}(I, H)}^{k-1}$, so $\left\{f_{n}(t)\right\}_{n \geq 1} \subseteq X^{*}$ is uniformly integrable. So, by the Dunford-Pettis theorem and by passing to a subsequence if necessary, we may assume that $f_{n} \rightarrow f$ weakly in $X^{*}$. Therefore, we obtain $\dot{x}+A(x)+B x=f, f \in F(t, x)$ a.e. on $I$. Since $x_{n}(t) \rightarrow x(t)$ in $L_{p}(I, H)$ and $\varphi: L_{p}(I, H) \rightarrow H$ is continuous, then we have

$$
x_{n}(0)=\left(1-\epsilon_{n}\right) \varphi\left(x_{n}\right) \rightarrow \varphi(x)=x(0) .
$$

Hence, evidently $x$ is a solution of (3.1). As in the proof of Theorem 3.1, we have that $|S|=\sup \left\{\|x\|_{W_{p q}}: x \in S\right\} \leq \hat{M}$, for some $\hat{M}>0$. So, $S \subseteq W_{p q}(I)$ is uniformly bounded. So, by the Dunford-Pettis theorem and by passing to a subsequence if necessary, we may assume that $x_{n} \rightarrow x$ weakly in $W_{p q}(I)$. As before, we have

$$
L_{\epsilon}(x)(t) \in \overline{\operatorname{conv}} \varlimsup\left\{L_{\epsilon} x_{n}(t)\right\}_{n \geq 1} \subseteq \overline{\operatorname{conv}} \varlimsup \lim F\left(t, x_{n}(t)\right) \subseteq F(t, x(t)) \quad \text { a.e. on } I \text {. }
$$

Clearly, $x(0)=\varphi(x)$, then $x \in S$. Thus, $S$ is weakly compact in $W_{p q}(I)$.

\section{Relaxation theorem}

Now, we prove the existence of extremal solutions and a strong relaxation theorem. Consider the extremal problem of the following evolution inclusion:

$$
\begin{aligned}
& \dot{x}+A(t, x)+B x \in \operatorname{ext} F(t, x) \quad \text { a.e. } I, \\
& x(0)=\varphi(x),
\end{aligned}
$$

where ext $F(t, x)$ denotes the extremal point set of $F(t, x)$. We need the following hypothesis:

(H5) $F: I \times H \rightarrow P_{w k c}(H)$ is a multifunction such that

(i) $(t, x) \rightarrow F(t, x)$ is graph measurable;

(ii) for almost all $t \in I, x \rightarrow F(t, x)$ is $h$-continuous; and (H2)(iii) holds.

Theorem 4.1 If hypotheses (H1), (H3) and (H5) hold, then the problem (4.1) has at least one solution.

Proof Since $S_{e} \subset S$, as in the proof of Theorem 3.1, we obtain a priori bound for $S_{e}$. We know that there exists $M_{i}>0, i=1,2$ such that $\|x\|_{W_{p q}}<M_{1}$ and $\|x\|_{C(I, H)}<M_{2}$ for all $x \in S_{e}$. Let $\psi(t)=b_{2}(t)+C_{5} M_{2}, \psi(t) \in L_{q}^{+}(I)$. We may assume that $|F(t, x)| \leq \psi(t)$ a.e. on $I$ for all $x \in H$. By Theorem 3.1, let $L_{0}=\dot{x}+A(x)+B x, x(0)=\varphi(x)$, then $L_{0}^{-1}: W_{p q}(I) \rightarrow X^{*}$ is well defined. So, let

$$
W=\left\{v \in L_{q}(I, H):\|v(t)\|_{H} \leq \psi(t) \text { a.e. on } I\right\},
$$

then $\hat{K}=L_{0}^{-1}(W) \subseteq W_{p q}(I)$ is a compact convex subset in $C(I, H)$. Obviously, $\hat{K}$ is convex. We only need to show the compactness. Let $\left\{x_{n}\right\}_{n \geq 1} \subset \hat{K}$, then there exists $h_{n} \in W$ such that $L_{0}\left(x_{n}\right)=h_{n}$, i.e., $\dot{x}_{n}=h_{n}-A\left(x_{n}\right)-B x_{n}$. By the definition of $W, W$ is uniformly bounded in $L_{q}(I, H)$. By the Dunford-Pettis theorem, passing to a subsequence if necessary, we may assume that $h_{n} \rightarrow h$ in $L_{q}(I, H)$ for some $h \in W$. From the definition of $W$, we have

$$
\left\|x_{n}\right\|_{W_{p q}}=\left\|L_{0}^{-1}\left(L_{0} x_{n}\right)\right\|_{W_{p q}}=\left\|L_{0}^{-1} h_{n}\right\|_{W_{p q}} \leq M_{1} .
$$


Therefore, the sequence $\left\{x_{n}\right\}_{n \geq 1} \subset W_{p q}(I)$ is bounded. Because of the compactness of the embedding $W_{p q}(I) \subset L_{p}(I, H)$, we have that the sequence $\left\{x_{n}\right\}_{n \geq 1} \subset L_{p}(I, H)$ is relatively compact. So, by passing to a subsequence if necessary, we may assume that $x_{n} \rightarrow x$ in $L_{p}(I, H)$. Moreover, by the boundedness of the sequence $\left\{x_{n}\right\}_{n \geq 1} \subset W_{p q}(I)$, it follows that the sequence $\left\{\dot{x}_{n}\right\}_{n \geq 1} \subset X^{*}$ is uniformly bounded and, passing to subsequence if necessary, we may assume that $\dot{x}_{n} \rightarrow \dot{x}$ in $X^{*}$. Since the embedding $W_{p q}(I) \subset C(I, H)$ is continuous and $W_{p q}(I) \subset L_{p}(I, H)$ is compact, it follows that $x_{n} \rightarrow x$ in $C(I, H)$ and $x_{n} \rightarrow x$ in $L_{p}(I, H)$. Hence, $x_{n} \rightarrow x$ in $H$ for all $t \in I \backslash \Lambda, m(\Lambda)=0$ ( $m$ being the Lebesgue measure on $R$ ). Since $A$ is hemicontinuous and monotone and $B$ is a continuous linear operator, thus $A\left(x_{n}\right) \rightarrow$ $A(x), B x_{n} \rightarrow B x$ in $X^{*}$ and as $n \rightarrow \infty$, we obtain $\dot{x}+A(x)+B x=h$ a.e. on $I$ and $x(0)=\varphi(x)$. Note that

$$
\dot{x}_{n}-\dot{x}+\left(A\left(t, x_{n}\right)-A(t, x)\right)=h_{n}-h-\left(B x_{n}-B x\right) .
$$

Taking the inner product above with $x_{n}-x$ and integrating from 0 to $T$, one can see that

$$
\begin{aligned}
& \int_{0}^{T}\left(A\left(t, x_{n}\right)-A(t, x), x_{n}-x\right) d t \\
& \quad=\int_{0}^{T}\left(h_{n}-h, x_{n}-x\right) d t-\int_{0}^{T}\left(B x_{n}-B x, x_{n}-x\right) d t-\int_{0}^{T}\left(\dot{x}_{n}-\dot{x}, x_{n}-x\right) d t \\
& \quad \leq \int_{0}^{T}\left(h_{n}-h, x_{n}-x\right) d t+\left\|x_{n}(0)-x(0)\right\|^{2} \\
& \quad=\int_{0}^{T}\left(h_{n}, x_{n}-x\right) d t-\int_{0}^{T}\left(h, x_{n}-x\right) d t+\left\|x_{n}(0)-x(0)\right\|^{2} \\
& \quad \leq \int_{0}^{T}\|\varphi\|_{H}\left\|x_{n}-x\right\|_{H} d t+\int_{0}^{T}\|h\|_{H}\left\|x_{n}-x\right\|_{H} d t+\left\|\varphi\left(x_{n}\right)-\varphi(x)\right\|^{2} \\
& \quad \leq 2 \int_{0}^{T}\|\varphi\|_{H}\left\|x_{n}-x\right\|_{H} d t+\left\|\varphi\left(x_{n}\right)-\varphi(x)\right\|^{2} \rightarrow 0 \quad \text { as } n \rightarrow \infty .
\end{aligned}
$$

By the hypothesis (H1)(iii), it follows that

$$
\int_{0}^{T}\left(A\left(t, x_{n}\right)-A(t, x), x_{n}-x\right) d t \geq C_{1} \int_{0}^{T}\left\|x_{n}-x\right\|_{H}^{p} d t \rightarrow 0 \quad \text { as } n \rightarrow \infty .
$$

So, we can find $\tau \in I \backslash \Lambda$ such that

$$
\left\|x_{n}(\tau)-x(\tau)\right\|_{H} \rightarrow 0 \quad \text { as } n \rightarrow \infty
$$

Using the integration by parts formula for functions in $W_{p q}(I)$ (see Zeidler [26], Proposition 23.23), for any $t \in I$, we have

$$
\begin{aligned}
\left\|x_{n}(t)-x(t)\right\|_{H}^{2}= & \left\|x_{n}(\tau)-x(\tau)\right\|_{H}^{2}+2 \int_{\tau}^{t}\left(\dot{x}_{n}(s)-\dot{x}(s), x_{n}(s)-x(s)\right) d s \\
= & \left\|x_{n}(\tau)-x(\tau)\right\|_{H}^{2}+2 \int_{\tau}^{t}\left(h_{n}(s)-h(s), x_{n}(s)-x(s)\right) d s \\
& -2 \int_{\tau}^{t}\left\langle A\left(x_{n}\right)(s)-A(x)(s), x_{n}(s)-x(s)\right\rangle d s
\end{aligned}
$$




$$
\begin{aligned}
& -2 \int_{\tau}^{t}\left\langle B x_{n}(s)-B x(s), x_{n}(s)-x(s)\right\rangle d s \\
\leq & \left\|x_{n}(\tau)-x(\tau)\right\|_{H}^{2}+2 \int_{0}^{T}\left(h_{n}(t)-h(t), x_{n}(t)-x(t)\right) d t \\
\leq & \left\|x_{n}(\tau)-x(\tau)\right\|_{H}^{2}+4 \int_{0}^{T}|\varphi(t)|\left\|x_{n}(t)-x(t)\right\|_{H} d t \\
\leq & \left\|x_{n}(\tau)-x(\tau)\right\|_{H}^{2}+4\|\varphi(t)\|_{L_{q}(I)}\left\|x_{n}(t)-x(t)\right\|_{L_{p}(I, H)} .
\end{aligned}
$$

By (4.5), we see that

$$
\max _{t \in I}\left\|x_{n}(t)-x(t)\right\|_{H} \rightarrow 0 \quad \text { as } n \rightarrow \infty
$$

So, $x_{n}(t) \rightarrow x(t)$ in $C(I, H)$. Since $x=L_{0}^{-1}(h)$ with $h \in W$, we conclude that $L_{0}^{-1}(W) \subseteq C(I, H)$ is compact. From Lemma 2.3, we can find a continuous map $f: \hat{K} \rightarrow L_{q}(I, H)$ such that $f(x)(t) \in \operatorname{ext} F(t, x(t))$ a.e. on $I$ for all $x \in \hat{K}$. Then $L_{0}^{-1} \circ f$ is a compact operator. On applying the Schauder fixed point theorem, there exists an $x \in \hat{K}$ such that $x=L_{0}^{-1} \circ f(x)$. This is a solution of (4.1), and so $S_{e} \neq \varnothing$ in $W_{p q}(I)$.

For the relation theorem of the problem (4.1), we need the following definition and hypotheses.

Definition 4.1 A Carathéodory function $\mu: I \times R^{+} \rightarrow R^{+}$is said to be a Kamke function if it is integrally bounded on the bounded sets, $\mu(t, 0) \equiv 0$ and the unique solution of the differential equation $\dot{s}(t)=\mu(t, s(t)), s(0)=0$ is $s(t) \equiv 0$.

(H6) For each $t \in I$, there exists a Kamke function $\mu: I \times R^{+} \rightarrow R^{+}$such that

$$
h\left(F\left(\cdot, x_{1}\right), F\left(\cdot, x_{2}\right)\right) \leq \mu\left(t,\left\|x_{1}-x_{2}\right\|_{H}^{2}\right) \quad \text { for all } x_{1}, x_{2} \in H
$$

and (H5) hold.

Theorem 4.2 If hypotheses (H1), (H3) and (H6) hold, then $\bar{S}_{e}=S$, where the closure is taken in $C(I, H)$.

Proof Let $x \in S$, then there exist $f \in L_{q}(I, H)$ and $f(x)(t) \in F(t, x(t))$ a.e. on $I$ such that

$$
\begin{aligned}
& \dot{x}(t)+A(t, x(t))+B x=f(t, x), \\
& x(0)=\varphi(x) .
\end{aligned}
$$

As before, let $W=\left\{v \in L_{q}(I, H):\|v\|_{H} \leq \psi(t)\right.$ a.e. on $\left.I\right\}$, then $\hat{K}=L_{0}^{-1}(W) \subseteq W_{p q}(I)$ is a compact convex subset in $C(I, H)$. For every $y \in \hat{K}$, we define the multifunction

$$
Q_{\epsilon}(t)=\left\{v \in F(t, y):(f-v, x-y) \leq \frac{1}{2} \mu\left(t,\|x-y\|_{H}^{2}\right)+\epsilon\right\} .
$$

Clearly, for every $t \in I, Q_{\epsilon}(t) \neq \emptyset$, and it is graph measurable. On applying Aumann's selection theorem, we get a measurable function $v: I \rightarrow H$ such that $v(t) \in Q_{\epsilon}(t)$ almost 
everywhere on $I$. So, we define the multifunction

$$
R_{\epsilon}(y)=\left\{v \in S_{F(\cdot, y)}:(f-v, x-y) \leq \frac{1}{2} \mu\left(t,\|x-y\|_{H}^{2}\right)+\epsilon\right\} .
$$

We see that $R_{\epsilon}: \hat{K} \rightarrow 2^{L_{q}(I, H)}$ has nonempty and decomposable values. It follows from Theorem 3 of [29] that $\overline{R_{\epsilon}(\cdot)}$ is LSC. Therefore, $y \rightarrow \overline{R_{\epsilon}(y)}$ is LSC and has closed and decomposable values. So, we apply Lemma 2.2 to get a continuous map $f_{\epsilon}: \hat{K} \rightarrow L_{q}(I, H)$ such that $f_{\epsilon}(y) \in \overline{R_{\epsilon}(y)}$ for all $y \in \hat{K}$. Invoking II-Theorem 8.31 of [22] (in [22, p.260]), we can find a continuous map $g_{\epsilon}: \hat{K} \rightarrow L_{q}(I, H)$ such that $g_{\epsilon}(y)(t) \in \operatorname{ext} F(t, y)$ almost everywhere on $I$, and $\left\|f_{\epsilon}(y)-g_{\epsilon}(y)\right\|_{w} \leq \epsilon$ for all $y \in \hat{K}$. Now, let $\epsilon \rightarrow 0$ and set $f_{\epsilon_{n}}=f_{\epsilon}, g_{\epsilon_{n}}=g_{\epsilon}$. Note that $\left\|g_{\epsilon_{n}}(y)\right\|_{H} \leq \psi(t)$ a.e. on $I$ with $\psi \in L_{q}(I)$, so we have $g_{\epsilon_{n}} \rightarrow f_{\epsilon_{n}}$ in $L_{q}(I, H)$. We consider the following problem:

$$
\begin{aligned}
& \dot{x}(t)+A(x)(t)+B x=g_{\epsilon_{n}}(x)(t), \\
& x(0)=\varphi(x),
\end{aligned}
$$

where $g_{\epsilon_{n}}(x) \in \operatorname{ext} R_{\epsilon}(x)$. We see that $L_{0}^{-1} g_{\epsilon_{n}}: \hat{K} \rightarrow \hat{K}$ is a compact operator and by the Schauder fixed point theorem, we obtain a solution $x_{\epsilon_{n}} \in S_{e} \subset W_{p q}(I)$ of (4.1). We see that the sequence $\left\{x_{\epsilon_{n}}\right\}_{n \geq 1} \subset \hat{K}$ is uniformly bounded. So, by passing to a subsequence if necessary, we may assume that $x_{\epsilon_{n}} \rightarrow \hat{x}$ in $W_{p q}(I)$. From the proof of Theorem 4.1, we know that $x_{\epsilon_{n}} \rightarrow \hat{x}$ in $C(I, H)$ and $\hat{x}(0)=\varphi(\hat{x})$. Note that $L_{0} x_{\epsilon_{n}}-L_{0} x=g_{\epsilon_{n}}\left(x_{\epsilon_{n}}\right)-f(x)$. So, we have that

$$
\begin{aligned}
& \left(\dot{x}_{\epsilon_{n}}(t)-\dot{x}(t), x_{\epsilon_{n}}(t)-x(t)\right)+\left(A\left(x_{\epsilon_{n}}\right)(t)-A(x)(t), x_{\epsilon_{n}}(t)-x(t)\right) \\
& \quad+\left(B x_{\epsilon_{n}}(t)-B x(t), x_{\epsilon_{n}}(t)-x(t)\right)=\left(g_{\epsilon_{n}}\left(x_{\epsilon_{n}}\right)(t)-f(x)(t), x_{\epsilon_{n}}(t)-x(t)\right) .
\end{aligned}
$$

However,

$$
\begin{aligned}
& \left(A\left(x_{\epsilon_{n}}\right)(t)-A(x)(t), x_{\epsilon_{n}}(t)-x(t)\right) \geq 0, \\
& \left(B x_{\epsilon_{n}}(t)-B x(t), x_{\epsilon_{n}}(t)-x(t)\right) \geq 0 \quad \text { a.e. } I .
\end{aligned}
$$

Then

$$
\begin{aligned}
\left(\dot{x}_{\epsilon_{n}}(t)-\dot{x}(t), x_{\epsilon_{n}}(t)-x(t)\right) \\
\leq\left(g_{\epsilon_{n}}\left(x_{\epsilon_{n}}\right)(t)-f(x)(t), x_{\epsilon_{n}}(t)-x(t)\right) \\
=\left(g_{\epsilon_{n}}\left(x_{\epsilon_{n}}\right)(t)-f_{\epsilon_{n}}\left(x_{\epsilon_{n}}\right)(t), x_{\epsilon_{n}}(t)-x(t)\right) \\
\quad+\left(f_{\epsilon_{n}}\left(x_{\epsilon_{n}}\right)(t)-f(x)(t), x_{\epsilon_{n}}(t)-x(t)\right) .
\end{aligned}
$$

By $g_{\epsilon_{n}} \rightarrow f_{\epsilon_{n}}$ in $L_{q}(I, H)$ and $x_{\epsilon_{n}} \rightarrow \hat{x}$ in $L_{p}(I, H)$, we have that

$$
\begin{aligned}
& \left(g_{\epsilon_{n}}\left(x_{\epsilon_{n}}\right)(t)-f_{\epsilon_{n}}\left(x_{\epsilon_{n}}\right)(t), x_{\epsilon_{n}}(t)-x(t)\right) \\
& =\left(g_{\epsilon_{n}}\left(x_{\epsilon_{n}}\right)(t)-f_{\epsilon_{n}}\left(x_{\epsilon_{n}}\right)(t), x_{\epsilon_{n}}(t)-\hat{x}(t)\right) \\
& \quad+\left(g_{\epsilon_{n}}\left(x_{\epsilon_{n}}\right)(t)-f_{\epsilon_{n}}\left(x_{\epsilon_{n}}\right)(t), \hat{x}(t)-x(t)\right) \rightarrow 0 \quad \text { a.e. } I .
\end{aligned}
$$


Hence, there exists a constant $N_{0}>0$, one has that

$$
\left|\left(g_{\epsilon_{n}}\left(x_{\epsilon_{n}}\right)(t)-f_{\epsilon_{n}}\left(x_{\epsilon_{n}}\right)(t), x_{\epsilon_{n}}(t)-x(t)\right)\right|<\epsilon
$$

as $n>N_{0}$. It follows that

$$
\begin{aligned}
\frac{1}{2} \frac{d}{d t} & \left\|x_{\epsilon_{n}}-x\right\|_{H}^{2} \\
= & \left(\dot{x_{\epsilon_{n}}}(t)-\dot{x}(t), x_{\epsilon_{n}}(t)-x(t)\right) \\
\leq & \left(g_{\epsilon_{n}}\left(x_{\epsilon_{n}}\right)(t)-f_{\epsilon_{n}}\left(x_{\epsilon_{n}}\right)(t), x_{\epsilon_{n}}(t)-x(t)\right) \\
& +\left(f_{\epsilon_{n}}\left(x_{\epsilon_{n}}\right)(t)-f(x)(t), x_{\epsilon_{n}}(t)-x(t)\right) \\
\leq & \left(f_{\epsilon_{n}}\left(x_{\epsilon_{n}}\right)(t)-f(x)(t), x_{\epsilon_{n}}(t)-x(t)\right)+\epsilon \\
\leq & \frac{1}{2} \mu\left(t,\left\|x_{\epsilon_{n}}-x\right\|_{H}^{2}\right)+2 \epsilon .
\end{aligned}
$$

Hence, $\left\|x_{\epsilon}(t)-x(t)\right\|_{H}^{2} \leq Q(t)$, where $Q(0)=\left\|x_{\epsilon}(0)-x(0)\right\|_{H}^{2}$ and $\dot{Q}(t)=\mu(t, Q(t))+4 \epsilon$. By (4.7), then $Q(0)=0$. Let $\epsilon \rightarrow 0$, we have $\left\|x_{\epsilon}(t)-x(t)\right\|_{H} \rightarrow 0$. Therefore, $x=\hat{x}$, i.e., $x_{\epsilon} \rightarrow x$ and $x_{\epsilon} \in S_{e}$, and so $S \subseteq \overline{S_{e}}$. Also, $S$ is closed in $C(I, H)$ (see the proof of Theorem 3.2), thus $S=\overline{S_{e}}$.

\section{Examples}

As an application of the previous results, we introduce two examples. Let $\Omega$ be a bounded domain in $R^{N}$ with smooth boundary $\partial \Omega, T=[0, b], 0<b<\infty$. Firstly, consider the following nonlinear evolution equation with a discontinuous right-hand side:

$$
\begin{aligned}
& \frac{\partial u(t, x)}{\partial t}-\operatorname{div}\left(|\nabla u|^{p-2} \nabla u\right)+|u|^{p-2} u=f(t, x, u(t, x)) \quad \text { on } T \times \Omega, \\
& u(t, x)=0 \quad \text { on } T \times \partial \Omega \\
& u(0, x)=\frac{1}{2 b} \int_{0}^{b} u(s, x) d s+\frac{1}{2} u(b, x) \quad \text { on } \Omega .
\end{aligned}
$$

The $p$-Laplacian $\operatorname{div}\left(|\nabla u|^{p-2} \nabla u\right)$ arises in many applications such as Finsler geometry and non-Newtonian fluids. In [30], Liu showed the existence of anti-periodic solutions to the problem $(5.1)$ where $f(t, x, \cdot)$ is continuous.

Since $f(t, x, \cdot)$ is not continuous, the problem (5.1) need not have solutions. To obtain an existence theorem for (5.1), we pass to a multivalued problem by, roughly speaking, filling in the gaps at the discontinuity points of $f(t, x, \cdot)$. So, we introduce the functions $f_{1}(t, x, u)$ and $f_{2}(t, x, u)$ defined by

$$
f_{1}(t, x, u)=\underline{\lim }_{\xi \rightarrow u} f(t, x, \xi)=\sup _{\epsilon>0} \inf _{|\xi-u|<\epsilon} f(t, x, \xi)
$$

and

$$
f_{2}(t, x, u)=\varlimsup_{\xi \rightarrow u} f(t, x, \xi)=\inf _{\epsilon>0} \inf _{|\xi-u|<\epsilon} f(t, x, \xi)
$$


Set

$$
\hat{f}(t, x, u)=\left[f_{1}(t, x, u), f_{2}(t, x, u)\right]=\left\{v \in R: f_{1}(t, x, u) \leq v \leq f_{1}(t, x, u)\right\} .
$$

Then, instead of (5.1), we study the following multivalued nonlinear evolution inclusion:

$$
\begin{aligned}
& \frac{\partial u(t, x)}{\partial t}-\operatorname{div}\left(|\nabla u|^{p-2} \nabla u\right)+|u|^{p-2} u \in \hat{f}(t, x, u(t, x)) \quad \text { on } T \times \Omega, \\
& u(t, x)=0 \quad \text { on } T \times \partial \Omega \\
& u(0, x)=\frac{1}{2 b} \int_{0}^{b} u(s, x) d s+\frac{1}{2} u(b, x) \quad \text { on } \Omega .
\end{aligned}
$$

The hypotheses on the data of this problem (5.1) are the following:

(H7) (i) $f_{i}(t, x, u)(i=1,2)$ are Nemitsky-measurable, i.e., $u: T \times \Omega \rightarrow R$ for all measurable, $u \rightarrow f_{i}(t, x, u)(i=1,2)$ is measurable;

(ii) there exists $a_{2}(t) \in L^{q}(t)_{+}, C>0$, such that

$$
\left|f_{i}(t, x, u)\right| \leq a_{2}(t)+C\|u\|^{k-1},
$$

where $1 \leq k<p$.

In this case, the evolution triple is $V=W_{0}^{1, p}(\Omega), H=L^{2}(\Omega)$ and $V^{\prime \prime}=W^{-1, q}(\Omega)$. From the Sobolev embedding theorem, we see that all embeddings are compact. Let us define the following operator on $V$ :

$$
\langle A(u)(t), v\rangle=\int_{\Omega}\left(|\nabla u|^{p-2} \nabla u \cdot \nabla v+|u|^{p-2} u v\right) d x .
$$

By the monotone property of $p$-Laplacian, it is easy to verify that $A$ satisfies our hypothesis (H1). Let $F: T \times H \rightarrow P_{k c}(H)$ be defined by

$$
F(t, u)=\left\{g \in L^{2}(\Omega): f_{1}(t, x, u) \leq g(x) \leq f_{2}(t, x, u)\right\} .
$$

The hypothesis $(\mathrm{H} 7)$ implies that $(\mathrm{H} 4)$ is satisfied. Note that $f_{1}(t, x, \cdot)$ is lower semicontinuous, $f_{2}(t, x, \cdot)$ is upper semicontinuous, and so $\hat{f}(t, x, \cdot)$ is USC (see [22, Example 2.8, p.371]). Let $\varphi(u)=\frac{1}{2 b} \int_{0}^{b} u(s, x) d s+\frac{1}{2} u(b, x)$, it is easy to check that $\varphi$ satisfies our hypothesis (H3)(ii). Then, we rewrite equivalently (5.1) as (3.1) , with $A$ and $F$ as above. Finally, we can apply Theorem 3.2 to the problem (5.1) and obtain the following.

Theorem 5.1 If the hypothesis (H7) holds, then the problem (5.1) has a nonempty set of solutions $u \in L_{p}\left(T, W_{0}^{1, p}(\Omega)\right)$ such that $\frac{\partial u}{\partial t} \in L_{q}\left(T, W^{-1, p}(\Omega)\right)$.

Secondly, we present an example of a quasilinear distributed parameter control system, with $a$ priori feedback (i.e., state dependent control constraint set). So, let $T=[0, b]$ and $Z \subseteq R^{N}$ be a bounded domain with $C^{1}$-boundary $\Gamma$. Let $D_{k}=\frac{\partial}{\partial z_{k}}, k \in\{1,2, \ldots, N\}, \triangle=$ 
$\sum_{k=1}^{N} \frac{\partial^{2}}{\partial z_{k}^{2}}$. We consider the following control system:

$$
\begin{aligned}
& \frac{\partial x}{\partial t}-\sum_{k=1}^{N} D_{k} A_{k}(t, z, x, D x)-\triangle x=g(t, z, x(t, z)) u(t, z) \quad \text { a.e. on } T \times Z, \\
& \left.x\right|_{T \times \Gamma}=0, \quad x(0, z)=\frac{1}{b} \int_{0}^{b} x(s, z) d s \quad \text { a.e. on } Z, \\
& u(t, z) \in \operatorname{ext} U(t, z, x(t, z)) \quad \text { a.e. on } T \times Z .
\end{aligned}
$$

The hypotheses on the data (5.3) are the following:

(H8) $A_{k}(k=1,2, \ldots, N): T \times Z \times R \times R^{N} \rightarrow R$ are functions such that

(i) $(t, z) \rightarrow A_{k}(t, z, u, \eta)$ is measurable on $T \times Z$ for every $(u, \eta) \in R \times R^{N}$,

$(u, \eta) \rightarrow A_{k}(t, z, u, \eta)$ is continuous on $R \times R^{N}$ for all almost all $(t, z) \in T \times Z$;

(ii) $\left|A_{k}(t, z, u, \eta)\right| \leq \hat{\alpha}_{1}(t, z)+\hat{c}_{1}(z)(|u|+|\eta|)$ with a nonnegative function $\hat{\alpha}_{1} \in L^{2}(I \times Z)$ and $\hat{c}_{1}(z) \in L^{\infty}(Z)$ for almost all $t \in T$;

(iii) $\sum_{k=1}^{N}\left(A_{k}(t, z, u, \eta)-A_{k}\left(t, z, u, \eta^{\prime}\right)\right)\left(\eta_{k}-\eta_{k}^{\prime}\right) \geq\left|\eta-\eta^{\prime}\right|^{2}$ for almost all $t \in T$;

(iv) $A_{k}(t, z, 0,0)=0$ for all $(t, z) \in T \times Z$.

(H9) The function $g: T \times Z \times R \rightarrow R$ satisfies the following:

(i) for all $x \in R,(t, z) \rightarrow g(t, z, x)$ is measurable;

(ii) for all $(t, z) \in T \times Z, x \rightarrow g(t, z, x)$ is continuous;

(iii) for almost all $(t, z) \in T \times Z$ and all $x \in R$, we have

$$
|g(t, z, x)| \leq \eta_{1}(t, z)+\eta_{2}(t)|x|
$$

with $\eta_{1} \in L_{2}\left(T, L^{2}(Z)\right), \eta_{2} \in L^{\infty}(T)$.

(H10) $U: T \times Z \times R \rightarrow P_{k c}(R)$ is a multifunction such that

(i) for all $x \in R,(t, z) \rightarrow U(t, z, x)$ is measurable;

(ii) for all $(t, z) \in T \times Z, x \rightarrow U(t, z, x)$ is $h$-continuous;

(iii) for almost all $(t, z) \in T \times Z$ and all $x \in R,|U(t, z, x)| \leq \gamma$, with $\gamma>0$.

Let $V=H_{0}^{1}(Z), H=L^{2}(Z), V^{*}=H^{-1}(Z)$. Then $\left(V, H, V^{*}\right)$ is an evolution triple with compact embeddings. Let $A: T \times V \rightarrow V^{*}, B: V \rightarrow V^{*}$ be the operators defined by

$$
\begin{aligned}
& \langle A(t, u), v\rangle=\int_{\Omega} \sum_{k=1}^{N} A_{k}(t, x, u, D u) D_{k} v d x, \\
& \langle B(u), v\rangle=\int_{\Omega} \sum_{k=1}^{N} D_{k} u D_{k} v d x
\end{aligned}
$$

for all $v \in H_{0}^{1}(Z)$. Let $\varphi(u)=\frac{1}{b} \int_{0}^{b} u(s, z) d s$.

Evidently, using the hypothesis (H8) , it is straightforward to check that $A, B, \varphi$ satisfy hypotheses (H1), (H3). Also, let $F: T \times L^{2}(Z) \rightarrow P_{k c}\left(L^{2}(Z)\right)$ be defined by

$$
F(t, x)=\left\{y \in L^{2}(Z): y(z)=g(t, z, x(z)) u(z), u(z) \in \operatorname{ext} U(t, z, x(t)) \text { a.e. on } Z\right\}
$$

Using hypotheses (H9) and (H10), it is straightforward to check that $F$ satisfies the hypothesis (H5). 
Rewrite the problem (5.3) in the following equivalent evolution inclusion form:

$$
\begin{aligned}
& \frac{\partial x}{\partial t}+A(t, x(t))+B x \in \operatorname{ext} F(t, x) \quad \text { a.e. on } T, \\
& x(0)=\varphi(x) .
\end{aligned}
$$

It is easy to get the following theorem by applying Theorem 4.1 to the problem (5.3).

Theorem 5.2 If hypotheses (H8)-(H10) hold, then the problem (5.3) has one solution $x \in$ $L_{2}\left(T, H_{0}^{1}(Z)\right) \cap C\left(T, L^{2}(Z)\right)$ with $\frac{\partial x}{\partial t} \in L_{2}\left(T, H^{-1}(Z)\right)$.

\section{Competing interests}

The authors declare that they have no competing interests.

\section{Authors' contributions}

$J Z$ and YC carried out the main part of this manuscript. CY participated in the discussion and corrected the main theorem. FC provided all examples for our results. All authors read and approved the final manuscript.

\section{Author details}

${ }^{1}$ School of Aerospace Engineering, Beijing Institute of Technology, Beijing, 100081, People's Republic of China. ${ }^{2}$ Fundamental Department, Aviation University of Air Force, Changchun, 130022, People's Republic of China. ${ }^{3}$ Institute of Mathematics, Jilin University, Changchun, 130012, People's Republic of China.

\section{Acknowledgements}

The authors are in debt to the anonymous referees whose comments helped them to improve the final version of this paper. This work is partially supported by the National Natural Science Foundation of China (No. 11172036, 11171350, 10902125) and the Natural Science Foundation of Jilin Province Grants 201115133

Received: 23 October 2012 Accepted: 18 January 2013 Published: 5 February 2013

\section{References}

1. Byszewski, L, Lakshmikantham, $\mathrm{V}$ : Theorem about the existence and uniqueness of a solution of a nonlocal abstract Cauchy problem in a Banach space. Appl. Anal. 40, 11-19 (1990)

2. Byszewski, L: Theorems about the existence and uniqueness of solutions of semilinear evolution nonlocal Cauchy problems. J. Math. Anal. Appl. 162, 494-505 (1991)

3. Aizicovici, S, Lee, H: Nonlinear nonlocal Cauchy problems in Banach spaces. Appl. Math. Lett. 18, $401-407$ (2005)

4. Aizicovici, S, McKibben, M: Existence results for a class of abstract nonlocal Cauchy problems. Nonlinear Anal. 39, $649-668(2000)$

5. Aizicovici, S, Staicu, V: Multivalued evolution equations with nonlocal initial conditions in Banach spaces. Nonlinear Differ. Equ. Appl. 14, 361-376 (2007)

6. García-Falset, J: Existence results and asymptotic behaviour for nonlocal abstract Cauchy problems. J. Math. Anal. Appl. 338, 639-652 (2008)

7. García-Falset, J, Reich, S: Integral solutions to a class of nonlocal evolution equations. Commun. Contemp. Math. 12, 1031-1054 (2010)

8. Paicu, A, Vrabie, II: A class of nonlinear evolution equations subjected to nonlocal initial conditions. Nonlinear Anal. 72, 4091-4100 (2010)

9. Deng, K: Exponential decay of solutions of semilinear parabolic equations with initial boundary conditions. J. Math. Anal. Appl. 179, 630-637 (1993)

10. Ntouyas, S, Tsamatos, P: Global existence for semilinear evolution equations with nonlocal conditions. J. Math. Anal. Appl. 210, 679-687 (1997)

11. Byszewski, L, Akca, H: Existence of solutions of a semilinear functional-differential evolution nonlocal problem. Nonlinear Anal. 34, 65-72 (1998)

12. Fu, X, Ezzinbi, K: Existence of solutions for neutral functional differential evolution equations with nonlocal conditions. Nonlinear Anal. 54, 215-227 (2003)

13. Benchohra, M, Ntouyas, S: Nonlocal Cauchy problems for neutral functional differential and integrodifferential inclusions in Banach spaces. J. Math. Anal. Appl. 258, 573-590 (2001)

14. Kamenskii, M, Obukhovskii, V, Zecca, P: Condensing Multivalued Maps and Semilinear Differential Inclusions in Banach Spaces. de Gruyter Series in Nonlinear Anal. Appl., vol. 7. de Gruyter, Berlin (2001)

15. Liang, J, Liu, J, Xiao, T: Nonlocal Cauchy problems governed by compact operator families. Nonlinear Anal. 57 , 183-189 (2004)

16. Liang, J, Liu, J, Xiao, T: Nonlocal impulsive problems for nonlinear differential equations in Banach spaces. Math. Comput. Model. 49, 798-804 (2009)

17. Xue, X: Semilinear nonlocal problems without the assumptions of compactness in Banach spaces. Anal. Appl. 8 $211-225(2010)$ 
18. Xue, X: Nonlocal nonlinear differential equations with a measure of noncompactness in Banach spaces. Nonlinear Anal. 70, 2593-2601 (2009)

19. Tolstonogov, A: Extremal selections of multivalued mappings and the "bang-bang" principle for evolutions inclusions. Sov. Math. Dokl. 43(2), 481-485 (1991)

20. Xue, $X$, Cheng, Y: Existence of periodic solutions of nonlinear evolution inclusions in Banach spaces. Nonlinear Anal., Real World Appl. 11, 459-471 (2010)

21. Xue, X, Yu, J: Periodic solutions for semi-linear evolution inclusions. J. Math. Anal. Appl. 331, 1246-1262 (2007)

22. Hu, S, Papageorgiou, NS: Handbook of Multivalued Analysis: Vol. I Theory. Kluwer Academic, Dordrecht (1997)

23. Zeidler, E: Nonlinear Functional Analysis and Its Applications, vol. II. Springer, Berlin (1990)

24. Dugundji, J, Granas, A: Fixed Point Theory. Monogr. Matematyczne, vol. 123, pp. 9-31. PWN, Warsaw (1986)

25. Bressan, A, Colombo, G: Extensions and selection of maps with decomposable values. Stud. Math. 90, 69-86 (1988)

26. Tolstonogov, A: Continuous selectors of multivalued maps with closed, nonconvex, decomposable values. Russ. Acad. Sci. Sb. Math. 185, 121-142 (1996)

27. Hiai, F, Umegaki, H: Integrals, conditional expectations and martingales of multivalued functions. J. Multivar. Anal. 7, 149-182 (1977)

28. Papageorgiou, NS: Convergence theorems for Banach space valued integrable multifunctions. Int. J. Math. Sci. 10, 433-442 (1987)

29. Donchev, T: Qualitative properties of a class differential inclusions. Glas. Mat. 31(51), 269-276 (1996)

30. Liu, ZH: Anti-periodic solutions to nonlinear evolution equations. J. Funct. Anal. 258, 2026-2033 (2010)

doi:10.1186/1687-2770-2013-15

Cite this article as: Zhang et al.: Properties of the solutions set for a class of nonlinear evolution inclusions with nonlocal conditions. Boundary Value Problems 2013 2013:15.

\section{Submit your manuscript to a SpringerOpen ${ }^{\circ}$ journal and benefit from:}

- Convenient online submission

- Rigorous peer review

- Immediate publication on acceptance

- Open access: articles freely available online

- High visibility within the field

- Retaining the copyright to your article 